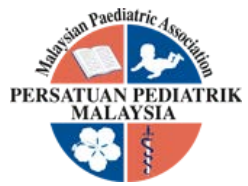

\title{
UNWITNESSED SNAKE BITE IN CHILDREN - A CHALLENGE IN DIAGNOSIS AND MANAGEMENT: A CASE REPORT
}

\author{
Lui Sze Yee, Noor Zuraini Abu Bakar, Ida Zaliza Zainol Abidin
}

\begin{abstract}
The clinical diagnosis of snakebite is critical, particularly in Southeast Asia where venomous snakebites are a public health concern. Additionally, cases involving unwitnessed snakebite with no species identification, especially in non-verbal children posed a challenge in the emergency setting. A 2-year-2-month-old boy presented to our emergency department with signs of neurotoxicity. He was restless and mildly bradypnoeic with the respiratory rate of 24 to 28 breaths per minute. He also had bilateral ptosis with absent gag reflex. There were faint fang marks noted over the medial aspect of his left ankle with local swelling and bruises, despite no history of animal bite and no eyewitness. A high index of suspicion of neurotoxic envenomation was prompted and a total of 6 vials of neuro-polyvalent anti-venom were administered in scheduled batches. Progressive clinical recovery was subsequently observed after the first batch of anti-venom administration. The case illustrated the importance of clinical recognition of neurotoxic envenomation in the absence of snake bite history or species identification. Early administration of anti-venom may potentially reverse the neurotoxic effects of systemic envenomation and saves lives.
\end{abstract}

\section{Keywords:}

Antivenom; Child; Respiratory Insufficiency; Snakebites; Neurotoxicity.

\section{Introduction}

The clinical diagnosis of snakebite is critical before any decision on the necessary management can be planned, particularly in the Southeast Asia where venomous snakebites are a public health concern. Cases involving unwitnessed snakebite with no species identification, especially in nonverbal children, hence posed as a challenge in the emergency setting.

Snakebites are commonly non-provocative in nature and there was a reported higher frequency of incidences during night times [1]. Lower limb was the commonest site of bites [1] as majority of snakebite cases are due to accidental stepping while walking or playing in the dark. Primary treatment includes the administration of antisnake venom and the tetanus toxoid.

There is various mode of clinical presentations in snakebite patients, which may either suggest a vasculotoxic or neuroparalytic nature of the
Received: 18 September 2019; Accepted revised manuscript: 13 November 2019

Published online: 03 December 2019

afflicted snakebite. In neuroparalytic bites, patients may present with altered sensorium, bilateral ptosis or complaining of diplopia within 8 hours of the bite event [1]. Progression of the systemic envenomation may result in respiratory failures, requiring ventilatory support. On the other hand, coagulation failure is the commonest complication following vasculotoxic snakebite, followed by hypotension [2].

There was a higher risk of fatalities in victims of snakebite who reported to the hospital after more than six hours of the bite incident, among which majority died within 10 hours of hospitalization [1].

\footnotetext{
Emergency and Trauma Department, Hospital Tuanku Fauziah, Perlis, Ministry of Health Malaysia

Corresponding author: Dr Lui Sze Yee,

Emergency and Trauma Department, Hospital Tuanku

Fauziah, Jalan Tun Abdul Razak, 01000 Kangar, Perlis, Malaysia.

Tel: +604-973 8000 Email: luiszeyee@hotmail.com
} 


\section{Case Report}

A 2-year-2-month old boy presented to our emergency department with signs of neurotoxicity. He was drowsy and restless. He was mildly bradypnoeic with the respiratory rate of 24 to 28 breaths per minute, narrowly escaping endotracheal intubation. He also had bilateral ptosis with absent gag reflex. There were faint bite marks noted over the medial aspect of his left ankle with local swelling and bruises, despite no history of animal bite and no eyewitness (Figure 1).

Lungs examination revealed bilateral crepitation and an urgent chest radiograph showed right- setting; the first two vials were given over 15 minutes, the subsequent two vials over an hour and the final two vials over an hour. Upon completion of the first batch of antivenom, the child's cough reflex improved, breathing was noted to be regular and the partial ptosis improved. His neurotoxic manifestations were resolved after completion of the antivenom administration and he was subsequently monitored in Paediatrics Intensive Care Unit.

Wound debridement over his left ankle was done under general anaesthesia on day 3 of admission, revealing a necrotizing fasciitis of the left ankle.

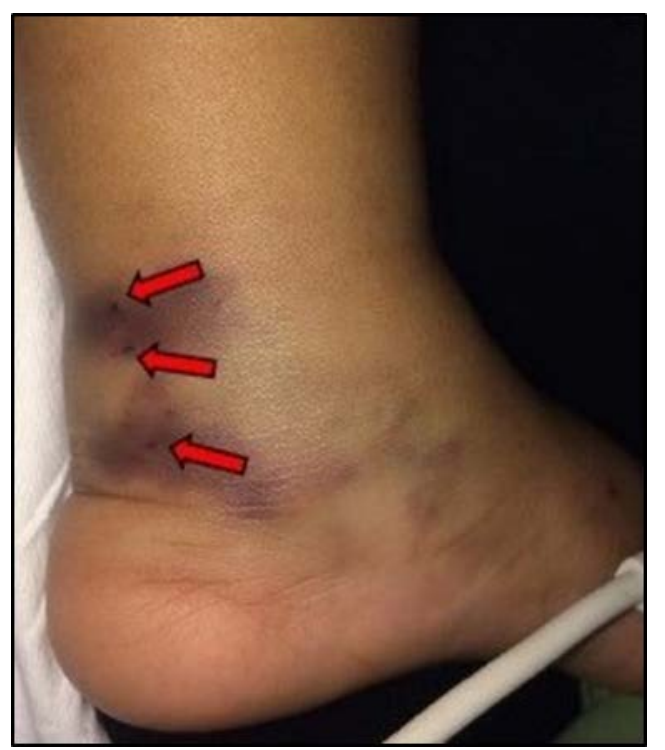

Figure 1 Preliminary case assessment in emergency department. Left leg - prominent bruises noted over the medial aspect of left ankle (near to the location of posterior tibial artery), localised swelling and necrotic patch with fang marks noted (red arrow).

sided patchy opacity, suggesting aspiration pneumonia. His baseline blood count revealed leucocytosis (TWCC: $18.04 \times 10^{3} / \mu \mathrm{L}$ ) with neutrophilic predominance and coagulation profile was within normal limits. Further questioning revealed that he had a prior visit to the local health clinic for left ankle swelling and was treated as an outpatient for soft tissue infection, prescribed with analgesia, before presenting to us with full blown neurotoxicity.

The clinical diagnosis of systemic snakebite envenomation was made based on the presenting clinical manifestation. A total of six neuropolyvalent snake antivenom (purified polyvalent equine immunoglobulin manufactured by Queen Saovabha Memorial Institute, Bangkok, Thailand) was administered in scheduled batches within the first hour of patient's arrival to our emergency
Intra-operative tissue culture and sensitivity revealed Morganella morganii, sensitive to cefuroxime and gentamicin. The wound gradually recovered with 10 days of high dose intravenous cefuroxime and regular wound dressing.

He was transferred out to general care on Day 4 of admission and was discharged well after 10 days of hospitalization.

\section{Discussion}

Snakebite cases in children were prevalent among boys older than 5 years old [1] due to their explorative tendency. Many children with toxicological emergencies resulting from systemic envenomation generally require non-invasive monitoring [3] however, our case illustrates the rare complication of necrotizing fasciitis in the affected limb requiring surgical wound debridement and intravenous antibiotics. 
There is currently no definite diagnostic marker for snakebite, and in the presence of insufficient information, further complicates the diagnosis of venomous snakebite in the clinical setting. Henceforth, meticulous observation of the clinical manifestations of snakebite is crucial in guiding the clinicians to develop a high index of suspicion of systemic envenomation. Apart from the localised signs and symptoms, severe cases may include general symptoms such as vomiting, central cyanosis, loss of consciousness, and systemic hypotension [3]. Our patient has had prior visit to the local health clinic with local signs of inflammation over the bite area but unfortunately was not suspected of having snakebite, which may be due to the progressive soft tissue changes masking the bite mark. Hence, the attending medical practitioner needs to embody a high index of suspicion when dealing with non-verbal children in the absence of eyewitness of the event.

Antivenom is an effective treatment for venomous snakebites. The monovalent anti-snake venom (ASV) specific to a particular snake species is the most appropriate therapy to be considered if there is positive species identification as targeted antivenom is associated with less anaphylactic reaction and is more effective in neutralising the venom [4]. Unfortunately in our case, in the absence of eyewitness of the bite event and no positive species identification, a neuro-polyvalent antivenom was administered instead with remarkable clinical results. This can be explained by cross-neutralization of heterologous venoms of closely-related species of the antivenins administered [5]. Additionally, the rationale behind the scheduled batches of antivenom administration in our paediatric patient was to guard against early anaphylactic reactions during the infusion. Apart from that, the application of tourniquets, incision and suction that were previously practiced are strongly discouraged due to its ineffectiveness and their potential in adding unnecessary risk to the injury [3].

Victims of venomous snakebites with significant wound injury should be closely monitored for secondary wound infections. Our patient required surgical wound debridement on Day 3 admission due to the progressive skin manifestation, revealing an intra-operative finding of necrotizing fasciitis over his left ankle. The risk factors for developing necrotizing fasciitis from cellulitis following snakebites were commonly associated with chronic underlying diseases and leukocytosis - a total white blood-cell counts of more than or equal to 10,000 cells $/ \mathrm{mm}^{3}$ with more than or equal to $80 \%$ of the white cells present in the segmented leukocyte forms [5].

Public awareness of snakebite cases should be regularly emphasized as the delay in seeking medical aid may result in substantial morbidity and mortality.

\section{Conclusion}

The case report illustrates the importance of clinical recognition of neurotoxic envenomation in the absence of snakebite history or species identification. The cluster of signs and symptoms present in this case underscores the rapidity of systemic envenomation and the diagnosis pitfall, that a clinician may mistaken a sign of neurotoxic paralysis for a drowsy child. Early administration of anti-venom may potentially reverse the neurotoxic effects of systemic envenomation and saves lives.

Necrotizing fasciitis that develops from snakebites is a surgical emergency, necessitating an emergency surgical fasciotomy. Hence, the attending physicians should practice caution when assessing the possibility of necrotic soft tissue infections following a snakebite event.

\section{References}

[1] Meshram RM, Bokade CM, Merchant S, Bhongade S. Clinical profile and outcome of snake bite in children. International Journal of Contemporary Pediatrics. 2017 Apr 25;4(3):910-4.

[2] Srimannarayana J, Dutta TK, Sahai A, Badrinath S. Rational use of anti-snake venom (ASV): trial of various regimens in hemotoxic snake envenomation. JAPI. 2004 Oct;52:78993.

[3] Hifumi T, Sakai A, Kondo Y, Yamamoto A, Morine N, Ato M, Shibayama K, Umezawa K, Kiriu N, Kato $H$, Koido $Y$. Venomous snake bites: clinical diagnosis and treatment. Journal of Intensive Care. 2015 Dec;3(1):16.

[4] Kumaravel KS, Ganesh J. A study on the clinical profile of children with snake envenomation in a tertiary referral centre at Dharmapuri, Tamilnadu, India. International Journal of Research in Medical Sciences. 2017 Jan 2;4(6):2142-5.

[5] Tan $\mathrm{CH}$, Leong PK, Fung SY, Sim SM, Ponnudurai G, Ariaratnam C, Khomvilai S, 
Sitprija V, Tan NH. Cross neutralization of Hypnale hypnale (hump-nosed pit viper) venom by polyvalent and monovalent Malayan pit viper antivenoms in vitro and in a rodent model. Acta tropica. 2011 Feb 1;117(2):119-24.

[6] Tsai YH, Hsu WH, Huang KC, Yu PA, Chen CL, Kuo LT. Necrotizing fasciitis following venomous snakebites in a tertiary hospital of southwest Taiwan. International Journal of Infectious Diseases. 2017 Oct 1; 63:30-6. 